\title{
Equivalences and differences between the hydrological dynamical systems of water budget, travel time, response time, and tracer concentrations and the legacy of models' topology
}

\author{
Riccardo Rigon ${ }^{1,2}$ and Marialaura Bancheri ${ }^{3}$ \\ ${ }^{1}$ University of Trento \\ ${ }^{2}$ Università di Trento, Dipartimento di Ingegneria Civile, Ambientale e Meccanica \\ ${ }^{3}$ Institute for Agricultural and Forest Systems in the Mediterranean, National Research \\ Council,
}

May 14, 2020

\begin{abstract}
We present, by using previous results on extended Petri Nets, the relations of various hydrological dynamical systems $(\$ \backslash$ mathtt $\{$ HDSys $\} \$$ ) derived from the water budget $(\$ \backslash$ mathtt $\{$ DynWB $\} \$)$. Once $\$ \backslash$ mathtt $\{$ DynWB $\} \$$ has been implemented, there exist a consistent way to get the equations for backward travel time distributions ( $\$ \backslash$ mathtt $\{\mathrm{DynTT}\} \$$ ), for the forward response time distribution $(\$ \backslash$ mathtt $\{$ DynRTD $\$)$ and for the concentration for a solute or a tracer $(\$ \backslash$ mathtt $\{$ DynC $\} \$)$. We show that the $\$ \backslash$ mathtt $\{$ DynWB $\} \$$ has a correspondence one to many with the $\$ \backslash$ mathtt $\{$ DynTT $\} \$$. In fact to any one of the $\$ \backslash$ mathtt $\{$ DynWB $\} \$$ equation correspond as many equation as the input precipitation events times. The $\$ \backslash$ mathtt $\{$ DynTT $\} \$$ is related to $\$ \backslash$ mathtt $\{$ DynRTD $\} \$$ by the Niemi's relationship and, in presence of multiple, $\$ \mathrm{n} \$$ outputs, by the specification of $\$ \mathrm{n}-1 \$$ partition functions, which determine which fraction of water volume, injected in the control volume at a specific time $\$ \mathrm{t}_{-}\{$in $\}$, goes asymptotically into a specific output. The $\$ \backslash$ mathtt $\{$ DynC $\}$, given $\$ \backslash$ mathtt $\{$ DynTT $\$$, depends further on the solute/tracer concentration in inputs. The paper clarifies the complicate set of relations above by using an example from literature. Upon the introduction of the appropriate information, it is also shown how these (\$ $\backslash$ mathtt $\{$ HDSys $\}$ ) can be solved simultaneously without duplicating calculations. It is also shown that these systems can be solved exactly, under the hypothesis of uniform mixing of water ages inside each reservoir within the system.
\end{abstract}

\section{Hosted file}

EquivalenceHYDs_HYP.pdf available at https://authorea.com/users/24891/articles/451123equivalences-and-differences-between-the-hydrological-dynamical-systems-of-water-budgettravel-time-response-time-and-tracer-concentrations-and-the-legacy-of-models-topology 


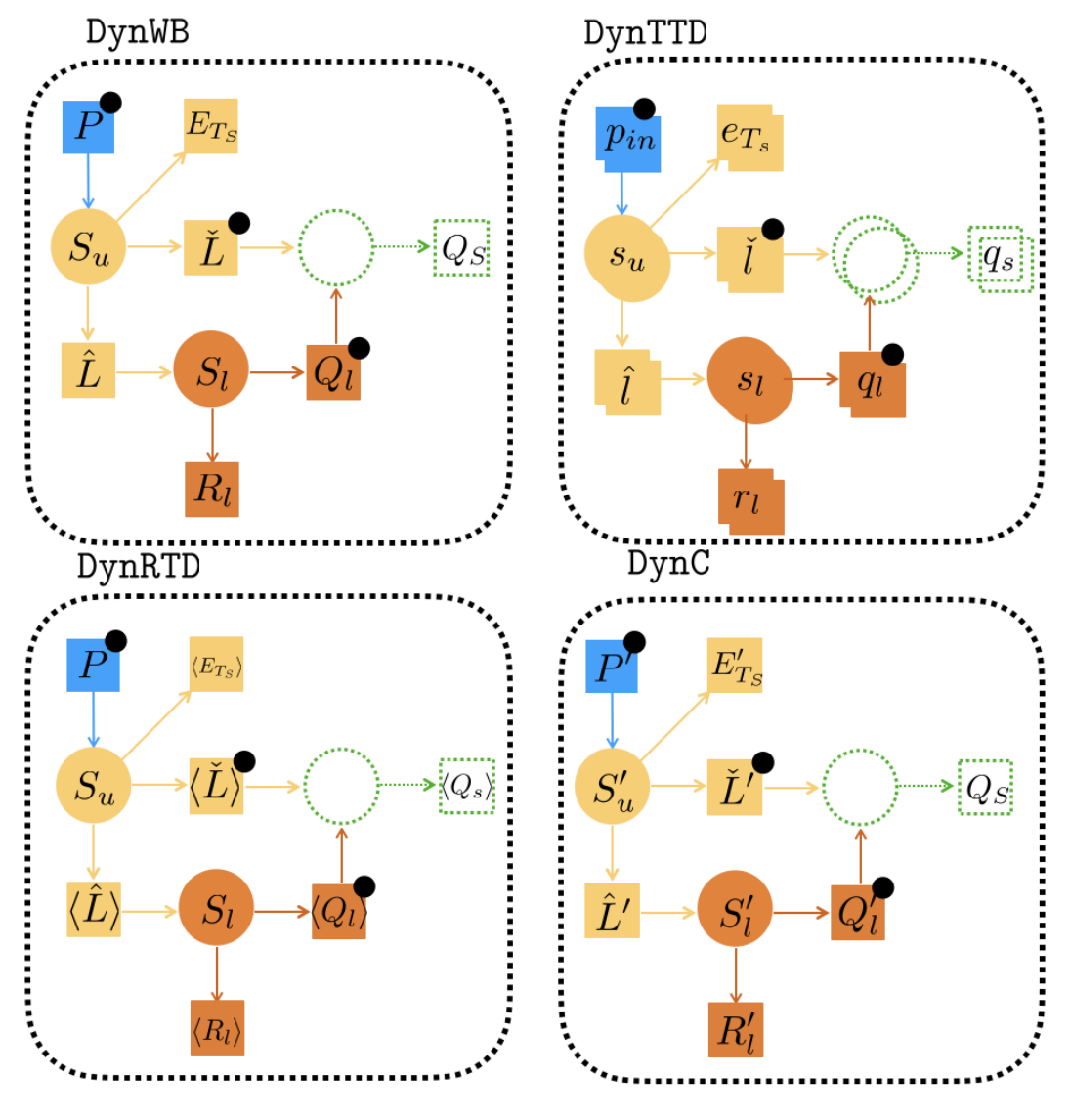

$\begin{array}{ll}U_{v} & \begin{array}{l}\text { place (reservoir) labeled with } \\ \text { state variable }\end{array} \\ & \begin{array}{l}\text { transition (flux) labeled } \\ \text { with variable }\end{array} \\ & \begin{array}{l}\text { controller labeled with } \\ \text { controlling variable }\end{array} \\ \text { generic arc }\end{array}$

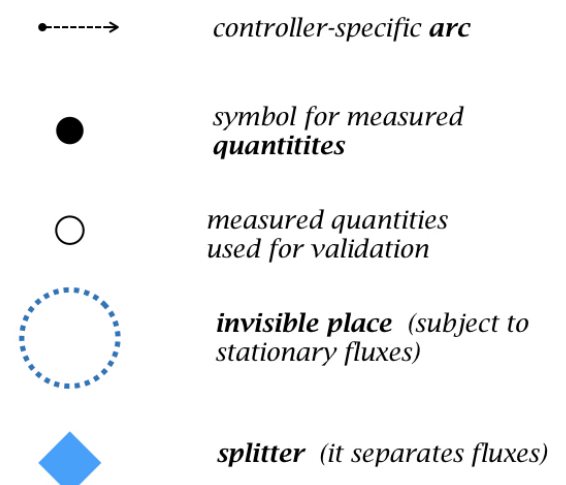

